\title{
Comparer les politiques agricoles américaines et européennes : les indicateurs ESP sont-ils bien utiles?
}

Comparing the US Farm Bill with the European Common Agricultural Policy: Are the PSE indicators really useful?

\section{Alexandre Gohin et Fabrice Levert}

\section{(2) OpenEdition}

Journals

Édition électronique

URL : http://journals.openedition.org/economierurale/956

DOI : 10.4000/economierurale.956

ISSN : 2105-2581

\section{Éditeur}

Société Française d'Économie Rurale (SFER)

Édition imprimée

Date de publication : 1 novembre 2006

Pagination : 92-106

ISSN : 0013-0559

Référence électronique

Alexandre Gohin et Fabrice Levert, «Comparer les politiques agricoles américaines et européennes : les indicateurs ESP sont-ils bien utiles? », Économie rurale [En ligne], 294-295 | Juillet-octobre 2006, mis en ligne le 29 octobre 2009, consulté le 19 avril 2019. URL : http://journals.openedition.org/ economierurale/956; DOI : 10.4000/economierurale.956 


\title{
Comparer les politiques agricoles américaines et européennes : les indicateurs ESP sont-ils bien utiles ?
}

\author{
Alexandre GOHIN • INRA ESR Rennes et Centre d'études prospectives et d'informations \\ internationales, CEPII
}

Fabrice LEVERT • INRA ESR Rennes

\section{Introduction}

D ébut 2007, les négociations commerciales multilatérales à l'Organisation mondiale du commerce (OMC) sont toujours bloquées, soit près de cinq années après leur lancement à Doha. Elles butent principalement sur le volet agricole où, de manière très schématique, l'Union européenne est accusée de ne pas vouloir ouvrir significativement son marché aux importations de produits agricoles et agroalimentaires, les États-Unis étant, quant à eux, accusés de ne pas vouloir réduire leurs soutiens internes.

Cette présentation très succincte de l'état des négociations à l'OMC est un révélateur intéressant des différences de politiques agricoles qui subsistent de part et d'autre de l'Atlantique. Cela ne signifie pas que ces différences sont aussi prononcées que par le passé. Par exemple, dans le secteur des grandes cultures, nous pouvons remarquer une même évolution de la Politique agricole commune (PAC) et des lois agricoles américaines vers une instrumentation dite plus découplée, c'est-à-dire où le soutien aux revenus agricoles est théoriquement moins lié aux volumes de production. Cette apparente convergence dans l'évolution des politiques agricoles européennes et nord américaines est cependant loin d'être totale. Toujours dans le secteur des grandes cultures, il est facile d'identifier les différences suivantes entre les deux politiques. Tout d'abord, sur le soutien direct au revenu agricole, les États-Unis ont réintroduit en 2002 des éléments de couplage dans leur politique agricole avec notamment les paiements anticycliques qui varient inversement avec les prix mondiaux. A contrario, les soutiens directs européens sont figés sur le plan budgétaire. Une autre source de différence majeure entre les deux politiques concerne la mise en jachère obligatoire pour maîtriser l'offre domestique. En 1996, les États-Unis ont abandonné cet outil de gestion des marchés alors qu'il était une des pierres angulaires des précédentes lois agricoles. À l'inverse, l'Union européenne a introduit depuis 1992 un gel obligatoire des terres qui varie autour du taux de $10 \%$ des surfaces totales en grandes cultures. Enfin, la gestion des marchés de grandes cultures est très différente dans l'esprit entre les deux entités. Du côté européen, les céréales bénéficient du régime de l'intervention, ce qui implique essentiellement des taxes à l'importation et des subventions directes à l'exportation; les graines oléagineuses européennes sont, elles, non protégées. Du côté américain, les marchés de toutes les grandes cultures sont régulés par des prix minima à la production (les loan rates) qui impliquent des subventions couplées à la production. Sur le plan commercial, les exportations américaines peuvent bénéficier des mécanismes de crédit à l'exportation et d'aide alimentaire.

Cette description très sommaire de quelques différences qui existent entre les politiques agricoles appliquées aux grandes cultures dans ces deux régions illustre leur complexité. Surtout, cela met en évidence que la comparaison de leurs effets sur les 
marchés et échanges mondiaux est très délicate alors qu'elle est cruciale pour faire avancer les négociations multilatérales. Face à cette difficulté, une solution logique pour les négociateurs est de s'appuyer sur les indicateurs de soutien agricole calculés par la direction Agriculture de l'Organisation de coopération et de développement économique (OCDE). Le plus utilisé est sans contexte l'indicateur de l'Estimation du soutien aux producteurs (ESP) défini comme étant la valeur monétaire des transferts aux producteurs agricoles qui résultent des mesures de politique agricole. Cet indicateur est calculé depuis 1986 et offre donc une information sur une longue période de l'évolution du soutien aux producteurs agricoles. Le tableau 1 ci-dessous fournit les évolutions des indicateurs ESP européens et américains sur l'ensemble des productions agricoles et également sur l'ensemble des productions de grandes cultures qui vont retenir notre attention dans cet article. Que cela soit pour la production agricole totale ou les grandes cultures seulement, l'image donnée par cet indicateur est claire : les transferts monétaires aux agriculteurs européens sont nettement plus importants que ceux accordés aux agriculteurs américains. L'impression est inchangée lorsque l'on utilise les ESP exprimés en pourcentage de la valeur de la production.

Les implications politiques de ces chiffres ne sont évidemment pas anodines. D'ailleurs, tant les négociateurs européens (Haniotis et Bascou, 2003 ; Lamy cité par Tangerman, $2004^{1}$; CE, 2006) que des acteurs agricoles français (Pluriagri, 2005) ${ }^{2}$ ont mis en garde contre une utilisation détournée de ceux-ci dans le cadre des débats internationaux.

1. Tangermann S. Farming Support: the Truth behin the Numbers. OECD Observer (2004, March). Available at www.oecdobserver.org/ news/ fullstory .php/aid.1223/Farming support:the_truth.htlm 2. Pluagri (2005). L'estimation du soutien aux agriculteurs agricoles de l'OCDE : un indicateur à manier avec précaution. Papier disponible à www.agpb.fr/fr/dossier/aide/20050707.asp
D'une manière générale, les critiques portent sur le fait que l'indicateur ESP agrège les transferts monétaires de différentes mesures de politique agricole qui n'ont pas forcément les mêmes incidences sur les marchés et échanges agricoles. Elles portent aussi sur le fait que ces montants ne correspondent pas aux gains potentiels à attendre d'une libéralisation totale des échanges agricoles qui pourraient se négocier dans les accords OMC. En fait, force est de reconnaitre que ces critiques ne sont pas fondamentalement nouvelles (Peters, 1988 ; Masters, 1993 ; Byerlee et Morris, 1993 ; Hertel, 1989a et 1989b). Plus récemment et dans le cadre des actuelles négociations agricoles à l'OMC, des économistes canadiens (Doyon et al, 2002), suisses (Rieder et al, 2003) ${ }^{3}$ et néerlandais (Oskam et Meester, 2006 ; Keyzer, 2006 ${ }^{4}$ ) ont repris ou émis de nouvelles critiques à l'utilisation ou la définition de ces ESP. En fait, la réponse à ces critiques nécessite de réaliser que les ESP ne sont pas une mesure des effets des politiques agricoles mais plutôt une mesure de l'effort des gouvernements respectifs pour soutenir leur secteur agricole. Le recours à des modèles de simulation est alors nécessaire si l'objectif est de mesurer ces effets.

L'objectif de cet article est justement de simuler et comparer les effets des politiques agricoles européennes et américaines sur les marchés et échanges mondiaux, et de les examiner par rapport au classement des ESP. En d'autres termes, la question est de savoir si, empiriquement, la hiérarchie des ESP est un bon indicateur pour les négociations actuelles sur ces deux politiques agricoles. La principale difficulté ici est de déterminer quel modèle de simulation utiliser, voire développer, pour mesurer ces effets. Il existe

3. Rieder P., Flury C., Guiliani G. Estimation du soutien à l'agriculture : alternative à la présentation traditionnelle de l'ESP. École polytechnique fédérale de Zurich, Institut d'économie rurale, Groupe des marchés politiques, document de travail, juin 2003. 4. Keyzer M. (2006). The PSE Once More. Amsterdam, Centre for World Food Studies. Available at www.sow.vu.nl/pdf/PSE_once_more.pdf 
en effet une multitude de façons de modéliser les marchés agricoles, chaque façon ayant potentiellement un impact sur la mesure absolue et relative des effets. En fait, cette multitude d'approches peut être réduite à deux familles de modèles avec, d'un côté, les modèles d'Équilibre général calculable (EGC) et de l'autre, les modèles d'équilibre partiel. Dans cet article, nous utilisons un représentant de chacune de ces deux familles pour accroître la robustesse de l'analyse. Il s'agit tout d'abord de la version « agricole » du modèle Global Trade Analysis Project (GTAP), modèle mondial d'EGC initialement développé à l'université de Purdue et très utilisé pour simuler les effets des différentes options de libéralisation dans le cadre des négociations actuelles à l'OMC. Il s'agit ensuite du modèle Oléosim initialement développé à l'INRA de Rennes, modèle mondial centré sur le secteur des grandes cultures et utilisé pour mesurer les effets mondiaux de la politique américaine aux grandes cultures. Ce deuxième modèle est de facture similaire à celui utilisé dans le récent panel « coton » ayant opposé les États-Unis au Brésil à l'OMC (Sumner, 2003). Ces deux modélisations sont utilisées ici pour simuler puis comparer les effets des politiques américaines et européennes aux grandes cultures sur les marchés mondiaux. Les simulations montrent dans les deux cas que la hiérarchie des effets sur les marchés mondiaux des principales grandes cultures dépend certes des produits (de manière non triviale) mais est généralement inverse à celle donnée par les ESP.

Cet article est structuré de la manière suivante. La première partie synthétise les principaux débats autour des ESP calculés par l'OCDE et formule également quelques commentaires additionnels sur la non prise en compte de certains instruments de politique agricole dans ces indicateurs. La seconde partie présente les principaux résultats des simulations conduites avec les deux modélisations des politiques agricoles.

Tableau 1. Évolution des estimations du soutien aux producteurs (ESP) européens et américains sur les productions agricoles et sur les grandes cultures (en monnaie nationale)

\begin{tabular}{|c|c|c|c|c|c|c|c|c|}
\hline & \multicolumn{4}{|c|}{ Toutes productions } & \multicolumn{4}{|c|}{ Grandes cultures } \\
\hline & \multicolumn{2}{|c|}{ Europe } & \multicolumn{2}{|c|}{ États-Unis } & \multicolumn{2}{|c|}{ Europe } & \multicolumn{2}{|c|}{ États-Unis } \\
\hline & $M €$ & $\%$ prod. & $M \$$ & $\%$ prod. & $M €$ & $\%$ prod. & $M \$$ & $\%$ prod. \\
\hline 1986 & 97371 & $44 \%$ & 38564 & $24 \%$ & 19820 & $56 \%$ & 18948 & $40 \%$ \\
\hline 1987 & 93176 & $42 \%$ & 39262 & $23 \%$ & 21170 & $60 \%$ & 18112 & $36 \%$ \\
\hline 1988 & 86376 & $38 \%$ & 31344 & $18 \%$ & 16892 & $45 \%$ & 11257 & $25 \%$ \\
\hline 1989 & 72326 & $29 \%$ & 39276 & $22 \%$ & 13354 & $35 \%$ & 9662 & $20 \%$ \\
\hline 1990 & 80946 & $33 \%$ & 31618 & $17 \%$ & 16829 & $46 \%$ & 10954 & $22 \%$ \\
\hline 1991 & 98803 & $39 \%$ & 30979 & $17 \%$ & 22748 & $55 \%$ & 10052 & $22 \%$ \\
\hline 1992 & 88219 & $35 \%$ & 31186 & $16 \%$ & 18271 & $52 \%$ & 10843 & $21 \%$ \\
\hline 1993 & 91628 & $38 \%$ & 33343 & $17 \%$ & 20605 & $55 \%$ & 11266 & $23 \%$ \\
\hline 1994 & 90180 & $36 \%$ & 29008 & $14 \%$ & 20992 & $55 \%$ & 10017 & $18 \%$ \\
\hline 1995 & 96779 & $36 \%$ & 20180 & $10 \%$ & 21208 & $49 \%$ & 4766 & $8 \%$ \\
\hline 1996 & 93199 & $33 \%$ & 28963 & $13 \%$ & 18559 & $38 \%$ & 7693 & $12 \%$ \\
\hline 1997 & 95318 & $34 \%$ & 29768 & $13 \%$ & 18674 & $40 \%$ & 8356 & $14 \%$ \\
\hline 1998 & 100917 & $34 \%$ & 46144 & $21 \%$ & 22952 & $51 \%$ & 15231 & $26 \%$ \\
\hline 1999 & 107173 & $37 \%$ & 55942 & $26 \%$ & 22689 & $53 \%$ & 20558 & $35 \%$ \\
\hline 2000 & 93338 & $39 \%$ & 53670 & $24 \%$ & 21948 & $46 \%$ & 21668 & $36 \%$ \\
\hline 2001 & 93061 & $33 \%$ & 51838 & $22 \%$ & 21804 & $47 \%$ & 17620 & $31 \%$ \\
\hline 2002 & 96989 & $32 \%$ & 39105 & $18 \%$ & 18897 & $42 \%$ & 12876 & $23 \%$ \\
\hline 2003 & 104474 & $34 \%$ & 35618 & $15 \%$ & 21337 & $47 \%$ & 10689 & $17 \%$ \\
\hline 2004 & 107686 & $36 \%$ & 46504 & $18 \%$ & 25096 & $41 \%$ & 18037 & $27 \%$ \\
\hline Moyenne 86-04 & 94103 & $36 \%$ & 37490 & $18 \%$ & 20202 & $48 \%$ & 13085 & $24 \%$ \\
\hline Moyenne 95-04 & 98893 & $35 \%$ & 40773 & $18 \%$ & 21316 & $45 \%$ & 13750 & $23 \%$ \\
\hline
\end{tabular}

Source : Calculs des auteurs, d'après l'OCDE 


\section{Les Estimations de soutien aux producteurs agricoles : une synthèse des débats}

\section{Définition}

L'idée de calculer des indicateurs de l'ampleur des politiques agricoles remonte à plus de trente ans (Josling, 1973). Sans entrer dans le détail des discussions initiales sur le choix des indicateurs et leur définition (voir, à ce propos, Peters, 1988 ou avec plus de recul, Legg, 2003), retenons tout de même le point fondamental suivant qui est à la source des débats : ses concepteurs ont voulu définir un agrégat simple à calculer, en dehors de toute modélisation, mais pouvant être ensuite intégré comme input des politiques agricoles dans des modélisations simulant leurs effets. D'ailleurs, initialement le sigle ESP signifiait Equivalent subvention à la production et les ESP devaient alimenter le modèle MTM de l'OCDE (qui a précédé l'actuel modèle Aglink de l'OCDE). Cette procédure en deux étapes a été probablement mal comprise en raison d'une définition malheureuse donnée initialement à ces ESP. En effet, ils étaient définis comme la perte de valeur ajoutée que subiraient les producteurs agricoles si leur politique agricole devait être supprimée alors que seule une modélisation peut éventuellement apporter une telle information. Cette ambiguité a donc suscité des confusions et des débats autour de l'utilité des ESP, dont on trouve encore aujourd'hui des traces dans, par exemple, Oskam et Meester (op. cit. $)^{5}$ Pourtant, l'OCDE a entrepris des efforts de clarification de ces indicateurs, les a amendés et complétés pour les rendre plus pertinents, suivant en cela les critiques émises dans ces papiers. Pour présenter de manière synthétique les principales critiques et les réponses apportées par l'OCDE, il est utile de donner la formule algébrique de ces ESP.

Pour un produit et un pays donné, l'ESP

5. À la page 126, les auteurs écrivent : "This suggests a $31 \%$ lower gross revenue level for farmers if all government support would be removed". total est calculé comme suit :

$$
E S P=\left(P_{d}-P_{m}\right) \cdot Q+B
$$

avec $P_{d}$ le prix domestique du produit, $P_{m}$ son prix mondial, $Q$ la production domestique et $B$ les soutiens budgétaires qui peuvent être décomposés en plusieurs catégories (essentiellement soutien budgétaire aux volumes produits, aux facteurs de production courants et historiques et aux inputs). Comme le fait apparaître cette simple formule, les ESP comprennent essentiellement deux parties avec d'abord le soutien des prix de marché donné par la différence entre prix domestique et prix mondial multipliée par la production domestique, et ensuite le soutien budgétaire, hors soutien des prix de marché.

Les critiques formulées jusqu'à présent sont nombreuses et diverses. Les deux critiques qui reviennent dans quasiment tous les articles portent sur l'additionnalité des transferts des différentes mesures de politique, c'est-à-dire sur le signe + entre les deux composantes dans la formule ci-dessus d'une part, et sur le prix mondial utilisé $P_{m}$, d'autre part. Nous expliquons tout d'abord ces deux critiques et les réponses apportées par l'OCDE. Puis nous ajoutons de nouvelles interrogations sur les mesures incluses dans ces indicateurs.

\section{Sur l'additionnalité des transferts}

Les ESP additionnent les valeurs monétaires des transferts opérés avec différents instruments de politique agricole. Un premier débat porte sur le fait que toutes ces mesures n'ont pas les mêmes effets sur les incitations de production. Cette critique nous paraît aujourd'hui résolue, au moins dans l'esprit, pour les raisons suivantes. Déjà, et même si cela peut paraître a priori cosmétique, l'OCDE a :

(i) revu la définition des ESP en explicitant plus clairement qu'il s'agit de transferts et non d'une évaluation ex ante des effets ;

(ii) changé la signification du sigle ESP qui veut dire maintenant Estimation du soutien aux producteurs ; 
(iii) détaillé et insisté sur l'importance de la composition de ces ESP.

Surtout, l'OCDE conduit en parallèle d'autres travaux qui visent plus précisément à simuler les effets des politiques agricoles avec des modèles qui peuvent reposer sur ces ESP. Entrent tout particulièrement dans cette catégorie ceux menés autour de la modélisation Policy Evaluation Matrix (PEM) qui incluent ces ESP. Une publication très souvent citée dans les articles sur les politiques agricoles (Dewbre et al, 2001) montre justement que les différentes catégories des ESP n'ont pas les mêmes effets de distorsion sur les productions ou les échanges (ni sur l'efficacité de transfert d'ailleurs).

En fait, le point central à comprendre ici est que tout agrégat est défini par rapport à un objectif donné. Pour les ESP, il s'agit des transferts monétaires en faveur des producteurs agricoles, ce qui n'est pas directement lié aux effets de distorsion des politiques agricoles. D'autres agrégats sont définis dans la littérature pour mesurer plus précisément ces effets de distorsion. Mais ceuxci supposent la mobilisation d'un modèle de simulation de politique agricole alors que, rappelons-le, l'objectif des ESP est justement de faire un minimum d'hypothèses (quitte à ce qu'elles soient abruptes) pour son calcul.

Sur l'utilisation du prix mondial observé Lors du calcul du soutien par les prix de marché, il est nécessaire d'utiliser un prix mondial exprimé en monnaie nationale. Ceci a soulevé deux critiques majeures. D'une part, sans changement de politique agricole, ni des conditions du marché mondial du produit agricole en considération, une variation du taux de change peut conduire à une variation de l'ESP alors que la politique est inchangée. D'autre part, le prix mondial observé dépend en partie des politiques agricoles et est vraisemblablement biaisé à la baisse par rapport à une situation sans ces politiques. Il conviendrait alors d'utiliser un prix mondial d'équilibre sans ces politiques.
De nouveau, ces deux critiques nous semblent résolues, même si nous ajoutons un bémol à un des arguments apportés par l'OCDE. Sur le premier point, il est tout à fait logique que les ESP changent, même lorsque la politique agricole est inchangée. En effet, les ESP mesurent l'intensité de la politique et cette intensité varie avec les conditions sur les marchés. Sur le deuxième point et la proposition de simuler un prix mondial sans politique agricole, Tangermann $(2005)^{6}$ retourne deux arguments principaux :

- d'une part, il faut s'accorder sur le prix mondial «d'équilibre », ce qui renvoie à la question du choix de la modélisation utilisée ; - d'autre part, en supposant qu'un consensus se dégage sur la valeur de ce prix mondial d'équilibre, cela conduirait potentiellement à des ESP négatifs pour certains couples produits-pays, et in fine à ce que certains décideurs publics concluent, à tort, à la nécessité d'une intervention sur la politique agricole.

Cette deuxième argumentation n'est pas supportée par l'analyse micro-économique comme l'exemple suivant l'illustre. Prenons le cas d'un petit pays exportateur sur plusieurs marchés. Plusieurs d'entre eux se ferment à cause de la mise en place de mesures de limitation aux importations et aux exportations, si bien que le marché mondial résiduel devient nettement plus petit avec potentiellement un prix mondial inférieur au niveau initial sans politique agricole. Il n'est pas alors irréaliste de supposer que ce petit pays exportateur devienne en fait un grand acteur sur ce marché mondial réduit, auquel cas il peut avoir intérêt à taxer ses exportations pour profiter des termes de l'échange. Par conséquent, un ESP négatif pourrait avoir le mérite de signaler à un pays qu'il est de son propre intérêt de mettre en place une mesure de politique agricole.

6. Tangermann S. (2005) Is the Concept of the Producer Support Estimate in Need of Revision? OECD Publishing, OECD Food, Agriculture and Fisheries, Working papers, 1, doi:10.1787/845314770374, disponible à www.oecd.org/dataoecd/6/49/35091989.pdf 
Ce bémol conduit en fait à se poser la question de la cohérence économique des ESP que nous avons déjà tranchée auparavant : l'ESP est un agrégat économique défini par rapport à un objectif donné (mesure des transferts aux producteurs) et n'est en aucun cas une mesure des effets de ces politiques. Par extension il ne peut servir directement à une analyse de leur efficacité. Il n'est donc pas possible de conclure sur le bien fondé ou pas d'une politique à partir des ESP, comme le suggère le deuxième argument fourni par Tangermann (2005).

\section{2. À propos de l'exclusion de certaines mesures de politique agricole}

De nombreuses autres limites ont pu être émises à l'encontre des $\mathrm{ESP}^{7}$. Nous centrons ici la discussion sur l'exclusion de certaines mesures de politique agricole dans ces indicateurs. En effet, le soutien à un secteur économique donné peut être réalisé de différentes manières, et dans le cas de la construction d'un agrégat comme l'ESP, se pose naturellement la question des mesures à prendre en compte. Dans le cadre de la comparaison des politiques américaines et européennes aux grandes cultures dans l'optique des négociations $\mathrm{OMC}$, le traitement de deux types de mesure est largement discutable.

- Les mesures américaines de politique commerciale aux grandes cultures

Il s'agit tout d'abord des mesures commerciales américaines que sont les crédits à l'exportation et l'aide alimentaire extérieure. Considérons particulièrement le cas de l'aide alimentaire externe, le problème du soutien apporté par les crédits à l'exportation est expliqué dans Mervoyer et al, (2001). Sur le plan strictement économique, l'effet du soutien apporté par le mécanisme de l'aide

7. Pour une synthèse plus complète, voir Gohin A. et Levert F. (2006). Comparer les politiques agricoles américaines et européennes : les indicateurs ESP sont-ils bien utiles? Rennes, Inra économie, document de travail, disponible à www.rennes.inra.fr/ economiel alimentaire externe est loin d'être tranché et rares sont les évaluations quantitatives ; en fait, nous ne connaissons qu'une étude (au mieux) préliminaire du FAPRI (2001), où tout repose dans la modélisation sur d'arbitraires coefficients d'additionnalité à l'importation et à l'exportation. Le constat selon lequel l'importance des volumes américains d'aide alimentaire suit celui des stocks américains de céréales laisse penser à un effet de soutien des prix (Young et al, 2001) ${ }^{8}$. Mais cela n'en constitue pas une preuve formelle. En effet, l'aide alimentaire américaine externe peut être perçue comme une «subvention à l'exportation » permettant d'écouler les stocks américains mais sans nécessairement influer sur les prix producteurs potentiellement figés au niveau des taux de prêts à la commercialisation (loan rates). Par ailleurs, même dans le cas où les prix producteurs sont supérieurs à ces loan rates, cette aide alimentaire crée une demande nette au niveau mondial qui implique une augmentation du prix mondial profitant potentiellement à tous les producteurs et pas seulement aux agriculteurs américains. Selon ce raisonnement, il n'y a donc pas lieu de calculer un avantage quelconque pour ces producteurs dans les ESP car c'est un avantage partagé par tous les producteurs.

Cette analyse pose tout de même une difficulté majeure, à savoir comment expliquer l'attachement des producteurs et de l'administration américaine à cet instrument dans les négociations de l'OMC ? Avant de donner deux raisons, soulignons déjà que dans le cas où les prix mondiaux sont strictement supérieurs aux loan rates, les producteurs américains gagnent comme les autres producteurs dans ce raisonnement. Maintenant, lorsque les prix mondiaux sont inférieurs aux loan rates, une première justification de l'aide alimentaire tient au fait qu'il y a un décalage entre le versement de la subvention

\footnotetext{
8. Young L., Abbott P., Leetmaa S. (2001). Export Competition: Issues and Options in the Agricultural Negotiations. IATRC Commissionned paper 15 , available at: www.iatrcweb.org
} 
au titre du loan rate (le loan deficiency payment) et le moment où la production est effectivement vendue sur le marché mondial. Dans la majorité des cas, le loan deficiency payment est versé en début de campagne tandis que l'effet sur les prix mondiaux de l'aide alimentaire externe ne se réalise qu'en milieu de campagne, si bien que les producteurs peuvent «battre » le loan rate. Ces gains intra-campagnes peuvent être très conséquents et l'aide alimentaire externe y joue un rôle favorable (Wescott and Price, 2001). Une deuxième justification de l'aide alimentaire externe est plus « temporelle » : puisqu'elle permet de soutenir les prix mondiaux, l'ajustement du niveau du loan rate pour la campagne suivante sera moindre. En effet, dans les définitions des lois agricoles américaines jusqu'à la dernière en date de 2002, les niveaux des loan rate sont ajustés tous les ans en fonction de l'évolution des cours mondiaux : il y a donc un intérêt « futur » à des prix mondiaux plus élevés aujourd'hui mais qui ne seront pas nécessairement aussi élevés lors de la campagne suivante.

Sous ces deux mécanismes, intra et inter campagne, l'aide alimentaire externe offre un soutien aux producteurs américains, même lorsque les prix mondiaux sont inférieurs aux loan rates. Théoriquement, l'effet de transfert intracampagne est pris en compte dans les ESP à la rubrique subventions à la production. En revanche, l'effet intercampagne n'est pas nécessairement pris en compte si, lors de la campagne suivante, les prix mondiaux sont supérieurs aux loan rates (auquel cas les subventions à la production sont nulles). Il nous semble quand même que l'aide alimentaire externe, parce qu'elle favorise potentiellement les prix mondiaux et par suite les niveaux des loan rates de la campagne future, procure un soutien « différé » spécifique aux agriculteurs américains en augmentant l'espérance du soutien l'année suivante et en réduisant la variance des revenus futurs.

Cette analyse des effets de soutien de l'aide alimentaire externe est bien évidem- ment très théorique et nécessite d'être poursuivie empiriquement. Le point essentiel à retenir ici est qu'il existe théoriquement des mécanismes économiques par lesquels l'aide alimentaire externe procure un soutien spécifique aux agriculteurs américains, permettant par la même occasion de comprendre les positions des acteurs à son égard. Du coup, cette aide alimentaire externe doit pouvoir se refléter dans les ESP, ce qui n'est pas le cas aujourd'hui concrètement. La grande question est bien évidemment comment intégrer ce soutien qui transite par des mécanismes très compliqués. Nous suggérons qu'au minimum les dépenses budgétaires associées soient fournies dans les fichiers informatiques (Excel) retraçant les ESP. Nous reconnaissons que cela est très imparfait car, selon cette solution, l'aide alimentaire ne serait toujours pas dans le calcul final des ESP. Tout de même, elle aurait au moins le mérite de reconnaître leur existence dans la phase d'actualisation des bases ESP. En affichant plus clairement leur existence, cette solution inciterait par ailleurs les modélisateurs à développer des spécifications permettant d'identifier ces effets alors que ceci n'est pas le cas aujourd'hui (sans nécessairement de mauvaises intentions, voir ci-après sur la modélisation GTAP $^{9}$ ).

\section{- Le gel des terres européen}

Le deuxième type de mesures dont le traitement dans les ESP nous semble critiquable concerne les mesures de contrôle de l'offre, tout particulièrement la mesure européenne de gel obligatoire des terres pour bénéficier des soutiens budgétaires. Les dépenses budgétaires associées aux terres gelées sont bien incluses dans les ESP dans la catégorie de l'utilisation contrainte des inputs. En revanche, cela ne prend pas en compte le fait qu'il y a potentiellement une perte de revenu et de profit des producteurs car ils ne peuvent pas mettre en culture toutes leurs surfaces.

9. Pour Global Trade Analysis Project. 
L'exemple suivant en statique (c'est-àdire sans réaction des producteurs en termes d'offre et de demande, donc sans modélisation) montre que la non prise en compte du gel obligatoire peut conduire à tort à un soutien lorsque la mesure vient taxer en fait le secteur agricole. Supposons initialement un secteur agricole non soutenu (l'ESP initial est nul) produisant sur une surface $L^{0}$ à un coût de production $c$ constant à l'hectare et que les rendements à l'hectare (notés $r$ ) sont constants. Son profit et l'ESP initial sont alors :

$$
\pi^{0}=\left(P_{m}-c\right) \cdot r \cdot L^{0} ; E S P^{0}=0
$$

Supposons maintenant que la politique agricole consiste en un soutien budgétaire à l'hectare d'un montant $a_{1}$ sur les surfaces cultivées notées $L^{1}$ et $a_{2}$ sur les surfaces gelées de manière obligatoire. En supposant le cas d'un petit pays, le profit et l'ESP deviennent :

$$
\begin{gathered}
\pi^{1}=\left(P_{m}-c\right) \cdot r \cdot L^{1}+a_{1} \cdot L^{1}+a_{2} \cdot\left(L^{0}-L^{1}\right) ; \\
E S P^{1}=a_{1} \cdot\left(L^{1}+a_{2} \cdot\left(L^{0}-L^{1}\right)\right.
\end{gathered}
$$

La comparaison de ces deux formules montre sans ambiguïté que pour les ESP, cette politique agricole a pour effet de soutenir le secteur alors que la comparaison de deux expressions de profit montre qu'il est possible en fait que ce secteur soit taxé. C'est le cas lorsque :

$$
a_{1} \cdot L^{1}+a_{2} \cdot\left(L^{0}-L^{1}\right) \leq\left(P_{m}-c\right) \cdot r \cdot\left(L^{0}-L^{1}\right)
$$

Cette formule montre, comme l'intuition le suggère, que si le gel des terres est important alors que les aides directes à l'hectare sont faibles, le secteur peut être taxé par cette politique. Le terme de droite exprime le manque à gagner sur les surfaces gelées. Il conviendrait dès lors de retrancher ce terme dans la formule de ces $\mathrm{ESP}^{10}$.

De nouveau, nous reconnaissons que l'application de cette solution n'est pas immédiate car cela suppose de connaître les coûts de production. Il nous semble néanmoins qu'il est ici plus facile de l'adopter en s'appuyant sur les comptes économiques de l'agriculture pour fournir ces informations au niveau des pays de l'OCDE. Au passage, le soutien des prix de marché dans les secteurs animaux tient effectivement compte des surcoûts liés à l'alimentation animale et donc la solution suggérée est déjà mise en œuvre par ailleurs. Elle aurait par ailleurs le mérite de faire reconnaître dans les ESP l'existence des conditions attachées aux soutiens directs sans calcul bien complexe. Mais il doit être clair que cette solution ne trancherait pas les effets de distorsions, loin de là, car il faut pour cela une modélisation, ce que nous envisageons à présent.

\section{Estimations des effets de distorsion des politiques agricoles européenne et américaine}

Les ESP ne mesurent en aucun cas les effets de distorsion des politiques agricoles. Nous examinons à présent ces effets en les simulant tout d'abord avec la modélisation GTAP, puis avec la modélisation Oléosim. Dans les deux approches, nous décrivons rapidement les modèles, notamment la représentation des politiques agricoles, avant de donner les principaux résultats.

\section{L'approche GTAP}

Le cadre GTAP comprend tout d'abord une base de données qui met en cohérence tous les flux économiques de tous les pays sur un nombre conséquent de produits et services. $\mathrm{La}$ dernière base de données disponible correspond aux flux observés de l'année 2001. Les politiques agricoles représentées dans cette base sont donc celles qui prévalaient avant les dernières réformes, de 2002 pour les ÉtatsUnis et de 2003 pour l'Europe. Nous l'utilisons en utilisant une agrégation sectorielle et géographique adaptée à notre objectif. Ce cadre comprend ensuite un modèle EGC qui exploite ces données pour expliquer les

10. Cette simple formule peut être facilement étendue au cas où le soutien des prix de marché existe parallèlement au gel des terres et aux aides directes. 
comportements des agents économiques face à des évolutions de marchés ou de politique agricole. Dans cet article, nous allons globalement utiliser la dernière version mise au point par l'université de Purdue (Keeney et Hertel, 2005) $)^{11}$ qui est à la base de certains travaux de la Banque mondiale (voir notamment Hertel et Winters, 2005) ${ }^{12}$.

Plus précisément, au niveau de la modélisation des politiques agricoles, nous avons amélioré la représentation du système américain des taux de prêts à la commercialisation : nous avons tenu compte du fait que les États-Unis modifiaient les subventions unitaires à la production en fonction des prix à leur frontière (afin d'assurer un prix au moins égal à ces taux de prêts pour les agriculteurs américains). De la même manière, nous avons supposé que les subventions aux exportations européennes aux céréales permettaient de garantir les prix d'intervention pour les producteurs européens. En revanche, même si la modélisation des autres instruments de la politique agricole nous semble perfectible, nous la maintenons ici. Il est en effet intéressant de travailler avec une version assez proche du modèle tel qu'il est développé outre Atlantique et qui est largement utilisée ${ }^{13}$. Cette modélisation suppose que les aides américaines contracycliques et les subventions aux programmes d'assurance agricole ont les mêmes effets que les paiements fixes. Les deux programmes sont représentés par des subventions à la terre alors que dans les travaux de l'USDA par exemple (Young et al, 2001), ces subventions sont considérées comme des subventions couplées à la production. Ceci est donc de nature à minorer dans le cadre GTAP les

11. Keeney R., Hertel T. (2005). GTAP-Agr, $A$ Framework for Assessing the Implications of Multilateral Changes in Agricultural Policies. GTAP Technical Paper 24, disponible à www.gtap.org 12. Hertel T., Winters A. (2005). Poverty Impacts of a WTO Agreement. Washington DC, Forthcoming, Pre-publication, version at www.gtap.org/poverty 13. De la même manière, même si certaines données nous semblent « curieuses », nous les conservons. effets de distorsion de la politique américaine. Par ailleurs, cette modélisation GTAP ne prend pas en compte explicitement le gel des terres européen. Enfin, soulignons la modélisation des instruments de soutien des prix de marché : les subventions directes aux exportations européennes sont incluses alors que les mécanismes américains d'aide alimentaire et de crédit aux exportations sont exclues. Toutes ces hypothèses vont dans le sens d'une sous-évaluation relative des effets de distorsion de la politique américaine aux grandes cultures par rapport à ceux de la politique européenne.

Avec cet outil, nous avons conduit deux simulations. Il s'agit tout d'abord de la suppression de tous les instruments de la PAC appliqués aux secteurs des grandes cultures. Il s'agit ensuite de la suppression des instruments américains appliqués à ces secteurs. Examinons en particulier les impacts sur les marchés du blé (tableau 2). La suppression des instruments européens aux grandes cultures conduit à un net recul de la production européenne de blé $(10,8 \%)$. Alors que la consommation européenne change marginalement, cela implique un net recul du solde européen d'échanges de blé : ce solde passe d'un excédent de 359 millions de dollars à un déficit de 667 millions de dollars. En valeur, le principal bénéficiaire de ce recul européen est la région Reste du monde (moindre déficit de 558 millions de dollars), suivi par le Canada (augmentation de l'excédent de 132 millions de dollars). Les États-Unis ne bénéficient que marginalement dans la mesure où on observe un léger recul des subventions directes à la production au titre des prêts à la commercialisation. Le recul de la production européenne est donc compensé par des augmentations dans les autres régions, augmentation qui atteint $1,85 \%$ pour la région Reste du monde.

Si les États-Unis avaient supprimé leur politique agricole aux grandes cultures en 2001, alors la production américaine de blé aurait été $23,6 \%$ inférieure à celle observée cette année-là. 


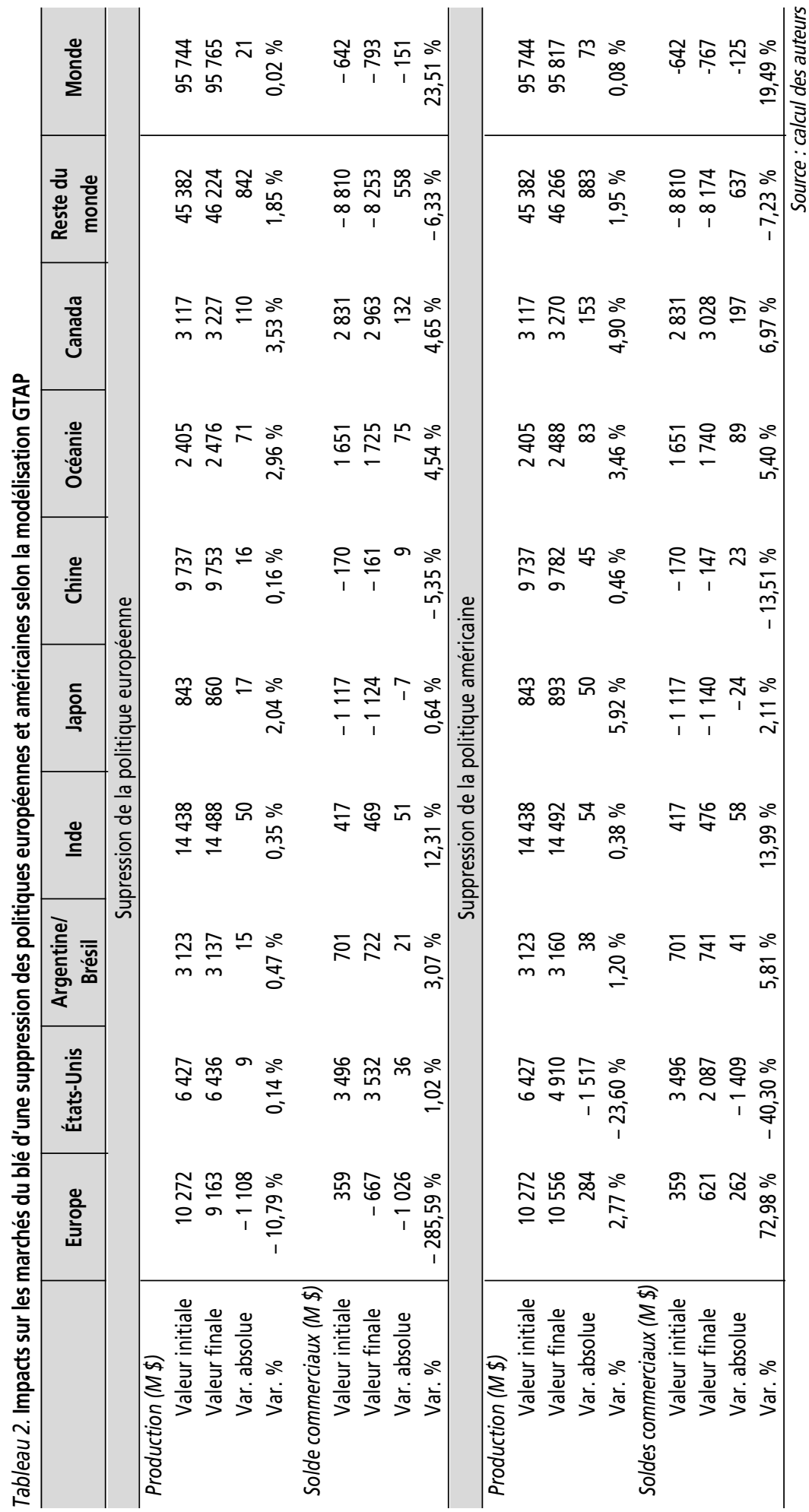


Là aussi, la consommation domestique est marginalement affectée par ce scénario, si bien que cela aurait entraîné un fort recul de l'excédent commercial américain en blé. Plus précisément, ce solde aurait reculé de 1409 millions de dollars mais serait resté positif. De manière symétrique, ce recul américain aurait d'abord profité à la région Reste du Monde, dont la production aurait augmenté de $1,95 \%$.

Il est notable de constater que les productions de blé dans toutes les régions du monde (exception faite bien sûr de l'UE et des EU) augmentent plus avec la suppression de la politique américaine qu'avec la suppression de la politique européenne, alors même que le soutien direct initial à cette production est plus fort en Europe (7,5 milliards de dollars) qu' aux États-Unis (4,3 milliards de dollars) dans les données GTAP basées sur les ESP. Deux raisons principales permettent d'expliquer cette apparente contradiction. D'une part, la composition du soutien n'est pas la même, avec l'UE ayant très peu (en termes relatifs) de soutien de marché ou de production. D'autre part, les effets croisés (des autres marchés et politiques) sont différents, la culture de blé étant une culture prépondérante en Europe et au contraire plus marginale aux États-Unis. Toujours est-il que ces impacts sur les marchés du blé montrent très clairement qu'il n'y a pas une simple relation linéaire entre l'importance du soutien public et les effets sur les marchés.

Examinons à présent les effets de ces deux simulations sur les profits des producteurs de grandes cultures dans les différentes régions (tableau 3).
Il apparaît que les politiques européennes et américaines aux grandes cultures ont des effets préjudiciables pour les producteurs de grandes cultures de toutes les autres zones. Plus précisément, en 2001, la politique américaine aux grandes cultures a eu pour effet de diminuer les profits des autres producteurs de grandes cultures de 5313 millions de dollars et la politique européenne de 4283 millions de dollars, quand bien même l'ESP européen calculé par l'OCDE pour les grandes cultures représente 1,35 fois celui de son homologue américain (18 648 millions de dollars). La distribution de ces effets dépend des pays, la politique européenne étant plus préjudiciable que la politique américaine pour le couple Argentine/Brésil et la Chine. À l'inverse, la politique américaine est nettement plus préjudiciable aux producteurs de la zone Reste du Monde (qui comprend en particulier l'Afrique) que la politique européenne. Soulignons finalement que la suppression de la politique européenne n'a que très peu d'effets sur le surplus des producteurs américains. À l'inverse, la suppression de la politique américaine conduit à une amélioration des profits des producteurs européens.

\section{L'approche Oléosim}

La comparaison des effets des politiques européennes et américaines aux grandes cultures est maintenant effectuée à partir du modèle Oléosim. Il s'agit d'un modèle d'équilibre partiel centré sur les secteurs des grandes cultures (notamment avec une bonne représentation du secteur des oléagineux) développé à l'initiative de la filière

Tableau 3. Impacts sur les surplus économiques des producteurs de grandes cultures de la suppression des politiques américaines et européennes selon les modélisations GTAP et Oléosim (M\$)

\begin{tabular}{l|c|c|c|c}
\hline \multicolumn{5}{c}{ Suppression de la politique européenne } \\
\hline & Europe & États-Unis & Autres & Pays Total \\
\hline Modélisation GTAP & -20503 & 11 & 4283 & -16209 \\
\hline Modélisation Oléosim & -16041 & -1 & 4643 & -11399 \\
\hline \multicolumn{7}{|c|}{ Suppression de la politique américaine } \\
\hline Modélisation GTAP & 564 & -15183 & 5313 & -9306 \\
\hline Modélisation Oléosim & 508 & -13368 & 6867 & -5993 \\
\hline
\end{tabular}


RECHERCHES

oléagineuse française dont les spécifications sont détaillées dans Gohin et Levert (op. cit. $)^{14}$ D'une manière générale, il s'agit d'un modèle de facture similaire aux principaux modèles d'équilibre partiel utilisés dans le cadre des négociations commerciales (modèle Aglink de l'OCDE, modèle ATPSM de la FAO, modèle FAPRI des universités américaines Iowa et Missouri).

D'une taille plus réduite, ce modèle peut être plus facilement mis en œuvre pour plusieurs années. Toutefois, dans une logique de comparaison, nous allons de nouveau nous concentrer sur les effets des politiques en 2001. Les données sur les marchés sont pour l'essentiel obtenues près de la base PSD (Production Supply Distribution) de l'USDA tandis que les données sur les politiques agricoles sont essentiellement obtenues auprès de la base ESP de l'OCDE. L'exception notable concerne le coton (non représenté dans cette base OCDE) et pour lequel nous avons utilisé des sources OMC (pour l'UE) ou de l'USDA (pour les EU). Au niveau de la modélisation proprement dite,

14. Cf. la note 7 . les élasticités prix sont reprises d'un modèle mis au point par l'USDA (SWOPSIM), et ont été actualisées pour l'année 2001. La représentation des instruments de politique agricole s'inspire de celle développée par Sumner (2003) dans l'étude qu'il a effectuée, à la demande du Brésil, dans le dossier coton opposant le Brésil aux EU. Plus précisément, dans les équations d'offre de grandes cultures, les instruments de soutien interne sont spécifiés avec la prise en compte de taux de couplage. Ces taux valent 1 pour les subventions à l'output et les subventions aux programmes d'assurance agricole, 0,25 pour les aides anticycliques et finalement 0,15 pour les aides directes fixes.

De nouveau, nous simulons d'abord une suppression de la politique européenne des grandes cultures, puis une suppression de la politique américaine. Les effets sur les marchés du blé et les prix mondiaux sont fournis dans les tableaux 4 et 5 . À titre d'exemple, il apparaît que la suppression de la PAC appliquée aux grandes cultures conduit à une diminution de la production européenne de blé de 4,4\% qui est partiellement compensée par une augmentation dans les autres régions. Au total, la produc-

Tableau 5. Impacts sur les prix mondiaux de la suppression des politiques européennes et américaines aux grandes cultures selon la modélisation Oléosim (\%)

\begin{tabular}{l|c|c}
\hline & $\begin{array}{c}\text { Suppression de la politique } \\
\text { européenne }\end{array}$ & $\begin{array}{c}\text { Suppression de la politique } \\
\text { américaine }\end{array}$ \\
\hline Blé & 2,10 & 2,59 \\
Orge & 3,13 & 2,35 \\
Maïs & 4,24 & 3,60 \\
Riz & 1,96 & 2,27 \\
Autres céréales & 6,15 & 3,19 \\
Graine de colza & 4,54 & 3,95 \\
Huile de colza & 4,61 & 3,21 \\
Tourteau de colza & 2,34 & 3,70 \\
Graine de tournesol & 3,94 & 3,62 \\
Huile de tournesol & 4,02 & 3,11 \\
Tourteau de tournesol & 1,22 & 3,54 \\
Graine de soja & 1,67 & 5,78 \\
Huile de soja & 3,61 & 4,50 \\
Tourteau de soja & 0,59 & 5,20 \\
Coton fibre & 0,42 & 9,67 \\
\hline
\end{tabular}


tion mondiale de blé recule en fait de $0,7 \%$ et le prix mondial augmente de $2,1 \%$. La suppression de l'intervention américaine en grandes cultures conduit de son côté à un recul de la production américaine de 5,8\%, de la production mondiale de blé de $0,3 \%$ et le prix mondial augmente de 2,6\%. L'effet prix mondial est donc plus fort avec la politique américaine, alors que le soutien direct américain est plus faible que le soutien direct européen pour cette culture du blé. Ceci est une nouvelle illustration qu'il n'y a pas une simple relation linéaire entre le soutien agricole et les effets sur les marchés mondiaux.

Les calculs des surplus économiques (voir le tableau 3) montrent, ici encore, que les États-Unis et l'Union européenne ont, par leur politique de soutien aux grandes cultures, un impact préjudiciable pour les producteurs de grandes cultures de toutes les autres zones. De nouveau, l'effet de la politique américaine est plus fort (6 867 millions de dollars) que celle de l'UE (4 643 millions de dollars).

\section{Conclusions}

Les négociations commerciales multilatérales (et même bilatérales) sont souvent difficiles à cause du dossier agricole. Dans le cadre de l'actuel cycle de négociations à l'OMC, les débats sur la PAC et la loi agricole américaine sont interminables car il s'agit de politiques très complexes. Les indicateurs ESP de l'OCDE fournissent une image de l'intensité de ces politiques et montrent que le soutien total est plus important en Europe qu' aux États-Unis. La question posée dans cet article est de savoir si ce classement ESP reflète bien les effets de ces politiques. Ces indicateurs ont été en effet longuement débattus car il s'agit d'agrégats définis en fonction d'un objectif donné et qui n'est pas celui de la mesure des effets de distorsion des politiques.

Dans ce texte, les différentes critiques portant sur les ESP ont été synthétisées et nous avons identifié également de nouvelles limites qui biaisent l'image donnée de l'intensité des politiques agricoles. En particulier, nous soulignons les faiblesses des actuels ESP dans la non prise en compte des mécanismes américains d'aides à l'exportation (aide alimentaire, crédit à l'exportation) d'une part, du mécanisme européen de gel obligatoire des terres d'autre part. Des solutions simples d'incorporation de l'intensité de ces instruments sont proposées. Surtout, nous simulons dans cet article les effets sur les marchés mondiaux des politiques européennes et américaines aux grandes cultures, avec deux modélisations utilisées ou utilisables dans les débats OMC. Nous trouvons que les effets de distorsion dépendent des marchés et des régions. Globalement, c'est-à-dire en termes d'effets sur les profits des producteurs de grandes cultures dans les autres régions, il apparaît que la politique américaine a plus d'effets négatifs que la politique européenne. Le classement obtenu avec ces deux modélisations est donc inverse à celui donné par les ESP.

Pour autant, nous pensons que les ESP sont utiles pour éclairer les débats sur les politiques et ce, pour deux raisons principales. D'une part, ces indicateurs fournissent une mesure « officielle » de l'importance de nombreuses mesures de politique agricole (comme toutes les subventions aux intrants) dans un cadre unifié, alors que les informations chiffrées sur ceux-ci sont sinon éparpillées. D'autre part, ces indicateurs sont régulièrement mis à jour, ce qui est sans contexte un avantage par rapport aux notifications des pays membres à l'OMC qui sont souvent très décalées dans le temps. Ils sont donc utiles pour apprécier l'évolution de l'importance des transferts statiques dus aux politiques agricoles. Toutefois, comme nous l'avons déjà souligné, ils peuvent être toujours améliorés dans cette optique de mesure de l'intensité des politiques par une couverture plus large de tous les instruments.

En revanche, nous ne sommes pas foncièrement favorables à ce que les ESP évoluent dans le sens d'une mesure des effets des 
politiques agricoles. Par exemple, il a été proposé par Rieder et al. (2003) $)^{15}$ et McClatchy $(1987)^{16}$ de pondérer les différentes composantes par leur degré de couplage. Cette solution, simple d'application et très peu coûteuse à mettre en œuvre, peut donc paraître séduisante à première vue. Cependant le choix des poids à accorder à chaque mesure est très problématique et fait implicitement recours à des résultats de modélisation. Par ailleurs, cette solution résout certes le problème de l'additionnalité évoqué dans cet article ; elle ne règle pas les autres problèmes, notamment celui de l'endogénéité des prix mondiaux et des quantités produites. Enfin, il n'est pas sûr qu'en termes de communication, cela ne soit pas source de confusion entre les ESP statiques et de nouveaux ESP «dynamiques ». À cet égard, soulignons que l'OCDE publie à côté des ESP totaux des ESP en pourcentage et des coefficients de protection nominaux dont nous n'avons pas fait mention ici car ils nous semblent relativement peu utilisés, même si l'OCDE fait l'effort de tous les présenter. Par conséquent, un indicateur supplémentaire peut avoir peu de chances d'être considéré, d'autant plus qu'il sera naturellement critiqué.

15. Cf. la note 3 .

16. Cité dans Cahill et Legg (1989).
Nous sommes plus favorables, au moins dans une optique de long terme, à des travaux effectués en parallèle sur la mesure des effets des politiques agricoles à partir de modélisations quantitatives. Cette suggestion est partagée par de nombreux économistes ${ }^{17}$ et peut être vue comme une application du principe de ciblage (à savoir un instrument par objectif). Bien entendu, cette solution n'est pas la plus facile à mettre en œuvre et est sujette à controverses car les résultats dépendent des spécifications dans les modèles. Dans cette étude, nous avons mobilisé deux modèles qui comportent des limites eux aussi. Ainsi, l'analyse est centrée sur les secteurs des grandes cultures, pour l'année 2001, en omettant les problèmes liés aux structures imparfaites de concurrence ou en ignorant les comportements d'aversion au risque des agents économiques. Mais force est de reconnaître qu'il s'agit-là de modélisations utilisées pour éclairer les actuels débats à l'OMC et qu'il vaut mieux porter les efforts sur leur amélioration dans la représentation de tous les instruments de politique agricole.

17. Les économistes de l'OCDE prônent naturellement l'utilisation de leur modèle PEM ; Oskam et Meester suggèrent, quant à eux, l'utilisation du modèle GTAP

\section{RÉFÉRENCES BIBLIOGRAPHIQUES}

Byerlee D., Morris M.-L. (1993). Calculating Levels of Protection: Is it always appropriate to use world reference prices based on current trading status? World development, 21(5), p. 805-815.

Cahill C., Legg W. (1989). Estimation of Agricultural Assistance Using Producer and Consumer Subsidy Equivalents: theory and practice. In OECD Economic Studies, Special Issue "Modelling the Effects of Agricultural Policies", 13, Winter 1989-1990.

Communauté européenne, CE (2006). Does the "Trade Talk" Match the "Trade
Walk"? Exploding the Myths Surrounding World Trade. Monitoring Agri-trade Policy, 03-06, 18 p.

Dewbre J., Anton J., Thompson W. (2001). The Transfer Efficiency and Trade Effects of Direct Payments. American Journal of Agricultural Economics, 83(5), p. 1204-1214.

Doyon M., Gouin D.-M., Paillat N. (2002). Analyse critique du concept d'ESP, estimation du soutien au producteur. Application au secteur laitier. Économie Rurale, $\mathrm{n}^{\circ} 272$, p. $74-87$. 
FAPRI (2001). Impacts of Additional Food Aid Allocations. FAPRI-UMC Report 15-01.

Haniotis T., Bascou P. (2003). The PSE: Is it reflecting or distorting, the trade impact of agricultural policies? Communication présentée lors de la conference internationale sur "Agricultural Policy Reform and the WTO: Where are we heading?", Capri, 23-26 June 2003.

Hertel T.-W. (1989a). Negotiating Reductions in Agricultural Support: Implications of technology and factor mobility. American Journal of Agricultural Economics, 71(3), p. 559-573.

Hertel T.-W. (1989b). PSEs and the Mix of Measures to Support Farm Incomes. World Economy, n ${ }^{\circ} 12.1$, p. 17-27.

Josling T.-E. (1973). Agricultural Protection: Domestic policy and international trade. Rome, FAO.

Legg W. (2003). Agricultural Subsidies: Measurements and use in policy evaluation. Presidential address. Journal of Agricultural Economics, 54(2), p. 175-200.

Masters W.-A. (1993). Measuring Protection in Agriculture: the producer subsidy equivalent revisited. Oxford Agrarian Studies, 21(2), p. 133-142.

McClatchy D. (1987). The Concept of Producer Subsidy Equivalent (PSE): Some Consideration with respect to International Negotiability. Agriculture Canada, Ottawa (May).
Mervoyer I., Beaumond H.-C., Kroll J.-C. (2001). Garanties et Soutiens publics aux Crédits à l'Exportation des Produits Agricoles aux États-Unis. Notes et Études Économiques, 14 , p. 9-35.

Oskam A., Meester G. (2006). How useful is the PSE in determining agricultural support? Food policy, 31, p. 123-141

Peters G.-H. (1988). The Interpretation and Use of Producer Subsidy Equivalents. Oxford Agrarian Studies, vol. XVII, p. 86-218

Sumner D.-A. (2003). Implications of the US Farm Bill of 2002 for Agricultural Trade and Trade Negotiations. Australian Journal of Agricultural Economics, 46(3), p. 99-122.

Tangermann S. (2003). À propos du concept d'ESP : commentaires et réponse. Économie rurale, $\mathrm{n}^{\circ}$. 276, p. 69-72.

Tangermann S. (2006). Response to the article on "How useful is the PSE in determining agricultural support?" by Arie Oskam and Gerrit Meester. Food policy, 31, p. 142-147

Westcott P., Price M. (2001). Analysis of the US commodity loan program with marketing loan provisions. USDA, Agricultural Economic Report 801, 26 p.

Young E., Vandeveer M.-L., Schnepf R.-D. (2001). Production and Price Impacts of Crop Insurance Programs. American Journal of Agricultural Economics, 83, p. $1192-1203$. 